\title{
Resignificar la relación pedagógica: dejar a la emoción fluir en el entre-nos
}

\author{
Resignifying the pedagogical relation: Let the emotion flow \\ in the in-between
}

\author{
Ángela María Cadavid Marín @ (1), Universidad de Manizales, Colombia. \\ Juan Pablo Parra Naranjo @ (1), Institución Educativa Pablo VI, Caldas, Colombia.
}

\begin{abstract}
Resumen
Indiscutiblemente la cotidianidad de la escuela, imbuida por lo parametrizado, lo mecanizado, lo instituido y lo normativo, imposibilitan miradas sensibles para reconocer la diferencia generacional entre maestros y estudiantes, y en lugar de ello dar paso al afecto. Se ha perdido todo rastro de sensibilidad en dicha relación, puesto que la mayoría de maestros crean barreras en los vínculos con sus estudiantes y se abandonan a los requerimientos que los generalizan y los homogenizan. Repensar esta relación no es una apuesta que surge de la nada, es un asunto que se manifiesta a partir de las voces de los estudiantes, quienes hacen evidente su postura, planteando, mediante sus relatos en la escuela, las formas como establecen vínculos o no con sus maestros a partir de las experiencias en los diversos espacios escolares en donde se movilizan y comparten con ellos gran parte de su tiempo. A partir de esto, los estudiantes proponen que la emoción y la afectividad son necesarias para revitalizar a la escuela de sensibilidad; en este sentido, del estudio realizado, aflora la necesidad de invitar al maestro a gestar espacios relacionales a la luz de una pedagogía sensible, para que, en lugar de generar desencuentros con el estudiante, entreteja con él vínculos de acogida, de respeto y de reconocimiento. Por tanto, con el presente artículo se pretende exhortar al maestro a resignificar la relación pedagógica desde una postura ética, a partir de la cual se reconozca al estudiante en su humana condición dejando a la emoción fluir en el entre-nos de esta relación.
\end{abstract}

Palabras claves: afectividad; emoción; relación pedagógica; relatos, sensibilidad; vínculo.

\begin{abstract}
School daily life which is undoubtedly imbued for what is parametrized, mechanized, instituted and regulated, makes the sensitive looks to recognize the generational difference between teachers and students impossible, and instead of it paves the way for affection. Any trace of sensibility has been lost in such relationship, since the majority of teachers build obstacles when bonding with their students and abandon themselves to the requirements that generalize and homogenize them. Rethinking this relationship is a bet that comes out of nowhere, is a matter manifested by the student's voices, who evidence their position, stating by means of their school stories, the ways in which they establish bonds or not with their teachers based on experiences in different schools spaces where they normally find themselves and spend a great deal of time with them. Therefore, the students suggest that the emotion and the affectivity are necessary to revitalize the school with sensitivity; for this matter, from the performed study, emerges the need to invite the teacher to promote relational spaces in terms of a sensitive pedagogy, so that, instead of causing misunderstandings with the student, the teacher interweaves with them welcoming bonds and also those of respect and recognition. Thus, it is intended with the present article to exhort the teacher to resignify the pedagogic relation from an ethical position, from which the student is recognized in its human condition letting the emotion flow in the in-between of this relation.
\end{abstract}

Keywords: affectivity; emotion; pedagogic relation; stories, sensitivity; bond. 


\section{Introducción ${ }^{1}$}

Estamos viviendo en un mundo donde las relaciones interpersonales se desmoronan con facilidad, debido a un sinnúmero de problemáticas sociales que ineludiblemente permean a las instituciones educativas. Los estudiantes cada vez están más inmersos en el conflicto social (la pertenencia a combos ${ }^{2}$ delincuenciales, los conflictos familiares, la desigualdad social, la falta de oportunidades, el consumo de drogas y alcohol, la violencia generalizada, entre otros), situación que se entremezcla con la vida escolar. Vivenciamos una realidad dentro de la escuela donde lo individual prospera por encima de lo colectivo, puesto que se denotan altos niveles de agresividad, de apatía, de indiferencia, de insolidaridad y de desconocimiento de la diversidad (Zemelman, 2005).

Frente a esto, una de las soluciones viables en la escuela, ha sido el carácter punitivo impuesto para resolver el conflicto que se vive en los diversos espacios o sobre aquellas conductas consideradas como inadecuadas; entre ellas, se destacan los llamados de atención, las anotaciones en cuadernos (observadores), que a manera de prontuarios recogen las acciones reprobables de los estudiantes, la citación a los padres en la institución, extrañamientos ${ }^{3}$ de la cotidianidad escolar y cuando definitivamente ninguno de estos correctivos funciona, se opta por la expulsión definitiva del estudiante; lo que hace parecer que ninguna de estas soluciones evita las problemáticas en la institución educativa.

Esto implica reconocer que los maestros habitamos en un espacio interpelado por momentos de crisis, lleno de cambios estructurales que implican nuevas lógicas y sensibilidades, nuevas demandas y exigencias que chocan contra los conocimientos impartidos, generando rupturas en la relación pedagógica; entendida esta, como aquella que propicia el vínculo entre maestro y estudiante, donde la práctica pedagógica se establece como una apuesta de responsabilidad en doble vía. Desde allí,

\footnotetext{
${ }^{1}$ Este artículo de carácter reflexivo y propositivo, surge a partir del Macroproyecto "Subjetividad, corporeidad y ciudadanía", adscrito a la Maestría en Educación dede la Diversidad (Centro Tutorial Manizales) de la Universidad de Manizales, con varios proyectos que lo soportaron (estudio realizado entre 2016-2018) y liderado por Angela María Cadavid Marín.

${ }^{2}$ Es un término que ha venido reemplazando paulatinamente al de pandilla.

3 Medida que se adopta a manera de sanción, cuando algún estudiante persiste en comportamientos considerados como inadecuados de acuerdo a los Manuales de Convivencia de las instituciones educativas. La medida opta por alejar al estudiante que comete una falta de la regularidad de las actividades académicas y de su grupo de compañeros.
} 
no solo se ha de buscar que el estudiante adquiera toda una serie de contenidos, sino que también interiorice virtudes y valores para aprender a convivir junto a otros (Aramburo y Vargas, 2015).

Así las cosas, vamos en contravía de una verdadera relación pedagógica de interacción / vínculo (Aramburo y Vargas, 2015, p. 29), puesto que venimos promulgando incesantemente la necesidad de alejarnos de los rezagos de la educación tradicional, pero seguimos olvidándonos del estudiante, concibiéndolo como un ser manipulable, a quien debemos procurarle una educación integral, la que hemos entendido como llenarlo de conocimientos y, además, disciplinarlo. Hemos desconocido el trasfondo real de la educación integral, cuyo propósito esencial es el de permitir el estar bien en todo momento y en todo lugar (Peralta, 2008); también posibilitar comodidad a los estudiantes "...con personas 'amorosas', en ambientes conocidos y en situaciones libres de estrés... rodeado de un ambiente afectuoso, consistente y que brinde seguridad" (p. 43).

De lo anterior, nos surgen varias inquietudes: ¿a qué educación integral hacemos referencia cuando olvidamos la afectividad y la emoción al querer compartir conocimiento?, ¿qué estamos haciendo frente a la ruptura de la relación pedagógica?, ¿cuál es la postura ética del maestro y de las instituciones educativas frente a estos acontecimientos?, ¿de qué manera estamos coadyuvando a acrecentar estas situaciones o a minimizarlas?

Todas estas preguntas parten de lo que evidenciamos dentro de las aulas, desconexión, ruptura y aislamiento con los estudiantes, debido, en la mayoría de los casos, a la verticalidad y autoritarismo que aún ejercen algunos maestros o a la normatividad que busca con castigos y sanciones dar solución a las problemáticas; lo único que se evidencia es que lo que se hace para solventar todo lo nombrado anteriormente, no logra acomodarse ni relacionarse con la realidad que se vive en la escuela, puesto que los conflictos continúan; ante esto, hacemos de tripas corazón y continuamos en nuestra cotidianidad, sobrellevando la situación.

Sin embargo, hemos querido hacer un alto en nuestra cotidianidad en la escuela y enfrentar esas inquietudes para reflexionar acerca de lo que experimentamos, ya que consideramos que tenemos una gran responsabilidad ética que nos lleva a tomar conciencia sobre el espacio donde estamos ubicados y el lugar que ocupamos en el devenir del estudiante; ese sujeto que nos ha confiado no solo una familia, sino también la sociedad. 
Tal reacción de querer enfrentarnos a las múltiples preguntas que nos hacemos, nos ha motivado a dar la palabra a los estudiantes y escuchar de su propia voz, sus posturas, propuestas y ángulos de realidad, alejando nuestra mirada adultocéntrica de maestros, en tanto consideramos que ha sido poco asertiva. Es decir, hemos decidido escuchar atentamente antes de tratar de oír en sus gritos, bullicios, carcajadas, Ilantos, indisciplina, la historia por construir.

Pensamos que es importante dejarnos sorprender por sus palabras y actos, mirándolos desde otra perspectiva, para leer, ya no desde la anormalidad de sus comportamientos el clamor a veces congelado por la institucionalidad, en demanda de mayor escucha, comprensión, libertad y posibilidad de ser espontáneos. De esa manera, brindamos espacios para que la expresión de sus pensamientos y sentimientos se dé de manera natural y nos permitan reconocer dónde se rompe ese fino hilo que dificulta generar vínculos para vivir armónicamente la escuela y de esa manera resignificar la relación pedagógica.

\section{Métodos y materiales}

Todo lo planteado en el primer apartado nos llevó a desarrollar un estudio con enfoque cualitativo, para comprender asuntos de la cotidianidad que van cobrando especial interés en la investigación en Ciencias Sociales; en este caso en particular, nuestro propósito gira alrededor de la percepción acerca de la realidad en el día a día de la vida escolar (Villegas y González, 2011), referida a la relación pedagógica desde la relevancia e importancia que le dan algunos estudiantes de varios municipios del Departamentos de Caldas para potenciar vínculos con los maestros.

Para dar cuenta de la percepción de la realidad por parte de los estudiantes, recurrimos al método biográfico narrativo, lo que nos permitió hacer una inmersión en la complejidad de las relaciones que se dan en los diferentes espacios escolares y dilucidar el significado que los estudiantes otorgan a esa realidad por medio de la narración de sus vivencias, donde les preguntamos sobre sucesos, acontecimientos, interacciones y pensamientos, que algunos de ellos han vivenciado en la escuela directamente con el maestro, en la búsqueda de respuestas a nuestras inquietudes para comprender cuáles son aquellos asuntos que dificultan la relación pedagógica y hace que se rompan los vínculos entre maestro y estudiante. 
Para cumplir con los anteriores postulados, los estudiantes participantes se escogieron de manera estratégica y de modo no aleatorio de acuerdo con varios criterios de selección; entre ellos, su participación voluntaria, su apertura para exponer pensamientos frente al tema investigado, su fluidez para expresarse y su disposición para abordar con postura crítica y reflexiva la información. Se ha de destacar que para este tipo de investigaciones el número de informantes es acotado, puesto que lo que se busca es realizar un análisis profundo a sus relatos de vida, que fueron los dispositivos utilizados para comprender la realidad escolar (Bolívar, Domingo y Fernández, 2001).

Al respecto del método biográfico narrativo, Álvarez (2012) expone que los dispositivos usados para que los sujetos testimonien su acontecer, permiten interpretar los diversos contextos en donde se han desenvuelto bajo su propia valoración personal, siendo relevante para los resultados del proceso de investigación en tiempos y espacios particulares; en tanto los informantes, en este caso los estudiantes, son quienes experimentan las situaciones directamente. Este método permite indagar

[...] en sus sensibilidades para dar cuenta de la comunicación y de la emergencia de un sujeto de la comunicación, sensible, expresivo que se junta para con-sentir, en la que los afectos, los sentimientos y las emociones se instalan como elementos con los que dotan de sentido y significado su existencia. (Cataño, 2017, p. 16)

Al tenor de lo anterior, quisimos "...habilitar la palabra y la escucha, así como los silencios y ocultamientos" (Jelin, 2014, p.142) del testimonio de los estudiantes, quienes contaron su experiencia por medio de entrevistas a profundidad a manera de conversatorios; lo que permitió mayor confianza para la expresión de pensamientos, imaginarios, sentimientos y acciones frente a acontecimientos de la cotidianidad escolar; en tanto “...es a través de los actos de habla explícitos por los que generalmente nos damos a entender. Estos permiten explicitar las subjetivaciones de nuestras propias vivencias frente a la objetividad de la realidad cotidiana" (Silvia, 2010, p. 113). Por tanto, nos dimos la oportunidad de escucharlos para entender la forma en que asumen su realidad, se posicionan y comportan en ella; porque están directamente involucrados en la problemática de la ruptura de la relación pedagógica.

Como se nombró previamente, los relatos de vida fueron los dispositivos escogidos para analizar la información recolectada durante los conversatorios realizados con los estudiantes. Estos aportaron a la configuración de los acontecimientos, dotando de sentido las vivencias que los participantes tienen en el mundo escolar, dando protagonismo a la significación que le dan a las mismas. 
Su aporte permitió complementar la mirada que como maestros teníamos de esta realidad, profundizando en la vida escolar desde las percepciones de los estudiantes quienes son los directamente implicados, enlazado esto con autores para desdoblar la información y construir conocimiento importante para reflexionar y dinamizar la cotidianidad en los diferentes espacios escolares. Para la interpretación de dicha información, se partió del análisis de los datos, para lo cual se usaron biogramas, los que permitieron dar

[...] un orden al conjunto de los sucesos pasados, encontrando un hilo conductor que establezca las relaciones necesarias entre lo que el narrador era y lo que hoy es; de esta manera, la narración media entre el pasado, presente y futuro, entre las experiencias acontecidas y el significado que ahora han adquirido para el narrador en relación a los proyectos futuros. (Bolívar et al., 2001, p. 193)

De esta manera, los biogramas posibilitaron llevar a cabo un análisis comprensivo de los relatos de vida, para dar paso a la interpretación desde categorías que emergieron y argumentar las posturas de los estudiantes con relación a la pregunta de investigación en los acontecimientos referidos con la relación pedagógica.

\section{Resultados}

Una vez analizada la información recolectada, evidenciamos claramente que las rupturas en la relación pedagógica se dan en varias direcciones; por tanto, hemos decidido utilizar un lenguaje que exhorte al maestro a reflexionar sobre su actuar a partir de las siguientes premisas:

- Gestar espacios relacionales para dotar a la escuela de una pedagogía sensible a partir de la emoción y la afectividad.

- Reconocer al estudiante en su humana condición dejando a la emoción fluir en el entre-nos de la relación pedagógica.

\subsection{Gestar espacios relacionales para dotar a la escuela de una pedagogía sensible a partir de la emoción y la afectividad}

Para los estudiantes es claro que sin emoción no se aprende; al respecto, Granada (2015) propone en su glosario de términos, que "Una emoción está siempre referida a una secuencia de acciones y los sentimientos se refieren a los resultados de esa secuencia, por lo que las emociones son unos programas de acción" (p. 5); por tanto, para los estudiantes es importante que en la relación pedagógica el maestro interactúe desde la provocación, pero que esta sea dada no por la información que 
brinde con base en sus conocimientos y saberes, sino por lo que termine pensando el estudiante; es decir, los efectos que se desencadenen al despertar su curiosidad por dicho saber, al provocar cosquillas mentales que inviten a reflexionar sobre la realidad y al ofrecerle la posibilidad de discernimiento repentino que los lleve a la resolución de problemáticas que se le presenten en su día a día.

Para los estudiantes al maestro le faltan más instrumentos para gestar espacios relacionales y de esta manera provocar el interés y la motivación por el aprendizaje; sienten que se valen de pocos elementos para motivarlos en las actividades del día a día, y que no consideran como "... imprescindible la motivación del estudiante para lograr el objetivo propuesto, pues sin motivación, será muy difícil hallar buenos resultados de apropiación del conocimiento y de la conciencia de aprendizaje" (Cadavid y Parra, 2010, p. 143).

En este sentido, los estudiantes manifiestan que los maestros utilizamos herramientas que son como dardos, pero que estos son lanzados suavemente y no provocan mayor reacción en ellos; el pensar y el actuar siguen siendo livianos, puesto que reciben muchos estímulos del exterior que los mantienen estáticos. En su mente hay un fuerte bullicio, una algarabía que no permite que su mensaje se incorpore. Algunos otros maestros no usan dardos, intentan con flechas; siendo más potentes, apenas consiguen rozar a los estudiantes, pero solo logran que unos pocos salgan del letargo académico.

En este sentido, están agotados de escuchar directrices basadas en el autoritarismo y quieren nadar en contracorriente complejizando aún más las situaciones en la escuela, simplemente porque sus gritos no son escuchados, sus manifestaciones no son reconocidas y sus actuaciones no permiten entrever la realidad que los envuelve y que de forma directa permea la escuela. La ausencia de cosquillas mentales los lleva a los actos de indisciplina porque no provocan mayor interés para la adquisición de conocimiento. Históricamente la relación pedagógica ha estado "...reducida a una orientación de tipo 'recetario', donde la instrumentalización homogeneizante reemplazó a la teoría y a la selección y creación curricular" (Peralta, 2008, p. 35).

La autora en mención nos plantea que muchas acciones del orden pedagógico no pasan por algún filtro, sino que se asumen con inmediatismos, sin realizar traducciones y adaptaciones, lo que ha llevado a que la pedagogía de la sensibilidad se haya debilitado y el estudiante se haya cosificado. Al respecto, Freinet (1973) expone "Si los educadores cobrasen conciencia del escaso rendimiento de su enseñanza, también verían la necesidad de modificar, de transformar su técnica» (p. 24); es 
decir, si continuamos en esta misma tónica siempre habrá ausencias y falencias que no nos dejen avanzar en la revitalización de la relación pedagógica; por ende, dotar a la escuela de sensibilidad, es preocuparnos por pedagogías intencionadas para despertar al estudiante y darle su lugar para que se interese y se involucre asertivamente en su proceso.

Cuando esto se logra, se reconoce el principio de individualidad (Peralta, 2008), visibilizando la singularidad y atendiendo la diversidad en el aula; lo que permite que los estudiantes reconozcan que el maestro tiene disposición; sin embargo, estos exponen en sus relatos que son muy pocos los maestros que persisten a pesar de no encontrar respuesta alguna, y que con su insistencia logran provocar esas cosquillas mentales que calan no solo en su mente, sino también en su corazón, seduciéndoles, encantándoles y conmoviéndoles. Al respecto, Goleman (1996) afirma que despertar la emoción no solamente humaniza la educación, sino que además invita y motiva al aprendizaje.

Por tanto, es imprescindible gestar esos espacios relacionales en donde surja la emoción y la afectividad para sacudir a los estudiantes y conquistarlos con sus pensamientos y acciones, evaporando de cierta manera el ruido ensordecedor de afuera; practicando una pedagogía sensible que invite y logre la participación activa y el interés por la adquisición del conocimiento revitalizando así a la escuela de sensibilidad; puesto "...que nuestra constitución como seres-en-el-mundo depende de nuestra relación con otros, y ésta no es sólo cognitiva (racional), sino también emocional" (García y Sabido, 2017, 669).

Estos maestros son reconocidos por sus estudiantes, consideran que van más allá y logran dejar huellas significativas, puesto que llegan a tatuar su alma con sus ideas, con sus miradas, generando vínculos y quitando el sabor amargo que produce la soledad que acompaña a muchos de ellos. El discurso del maestro en este sentido, logra cruzar esos mundos emotivos del estudiante, desperezándolos y despertándolos a la realidad para construir futuros posibles.

De modo que gestar espacios relacionales dotando a la escuela de una pedagogía sensible a partir de la emoción y la afectividad, nos permita adoptar posturas propias frente a todas esas mutaciones que alteran nuestra cotidianidad y que claman a gritos una resignificación de la relación pedagógica, lo que nos lleve a entender que debemos adaptarnos a las transformaciones de la sociedad, a ser capaces de interpretar las variaciones que se presentan en nuestro contexto y empecemos a 
asumir nuestra responsabilidad frente a los estudiantes, ubicándonos en un espacio - tiempo donde lo relacionado, lo habitual, lo acostumbrado y lo tradicional se quede relegado. En consonancia con este asunto, Bertoni (2015) expone

\begin{abstract}
También ha sido parte de nuestra experiencia el constatar una tendencia a tratar lo afectivo y lo vincular en el aula a partir de una matriz de seducción demagógica, que es una forma disfrazada del autoritarismo que caracteriza a la pedagogía tradicional. (p. 24)
\end{abstract}

Por tanto, avanzar en la aceptación y participación de una revitalización de nuestro papel, preocupándonos por ser más competentes para pensar, para sentir, para hacer y para dar a conocer nuestros pensamientos, dejando a la emoción fluir en el entre-nos, en una búsqueda consecuente de estrategias de vida que propendan porque lo concebido como ideal pase al mundo real, concientizándonos de la importancia de nuestro quehacer como maestros.

\title{
3.2 Reconocer al estudiante en su humana condición dejando a la emoción fluir en el entre-nos de la relación pedagógica
}

Los maestros seguimos desconociendo la diferencia, en donde aplicamos la universalidad y la serialización de manera restringida y excluyente dentro de los espacios escolares, alejando del mundo de la vida a aquellos estudiantes que no se encuadran allí, a tal punto que en nuestras aulas están llegando sujetos con poca cara de humanos porque seguimos nadando en medio del mar del analfabetismo afectivo, puesto que nos desligamos de la realidad vivida por los estudiantes.

Con esto en mente, no podemos ignorar que en los diversos espacios escolares confluyen estudiantes con diferentes características, variados intereses y además incontables emociones y por supuesto sensaciones. Si tuviéramos en cuenta todos estos aspectos, indudablemente la relación pedagógica potenciaría un vínculo más estrecho con el estudiante, pero además estaríamos ayudándolo a mantener buenas relaciones con los otros y con el contexto, ofreciéndole alternativas para empoderarse de una manera asertiva de los retos que enfrentaría más adelante en los ámbitos sociales como un sujeto capaz de contribuir a la transformación social; para Freire (2004), nuestra labor como maestros radica en aportar al devenir histórico del estudiante para que se convierta en protagonista de dicha transformación de la realidad a partir del conocimiento y de la acción.

A pesar de esto, los estudiantes en sus relatos de vida hacen referencia a que tal situación no se da y por el contrario, manifiestan la indiferencia por el sentir de los estudiantes y al mismo tiempo por hacer evidentes nuestras emociones, ya 
que en nuestro afán por impartir el conocimiento, cumplir con la multiplicidad y exceso de tareas antes de que se acabe la jornada, la neurosis que esto produce y además el control que ejerce el tiempo sobre nosotros, perdemos el equilibrio y escondemos nuestros sentimientos y también desconocemos en los estudiantes cuerpos congelados y hambrientos de afecto, ávidos de emociones y de aventura, condenados a contagiarse de nuestra desesperanza impregnada de pesimismo y desolación. En este sentido, es relevante traer a colación los aportes de Bedoya y González (2016), quienes invitan a retornar sobre "...los aspectos propios que subyacen a sus prácticas [del maestro] y estilos de vida, sus sentimientos y emociones que, en su mayoría, corresponden a una concepción centrada en el quehacer, en la ejecución de actividades propias del trabajo" (p.127).

En este sentido, los estudiantes manifiestan que la escuela nos quitó la sensibilidad, desvalorizó lo afectivo y nos deshumanizó, a tal punto que nuestro cuerpo no se atreve a dialogar con ellos; escondemos nuestras emociones para que el cuerpo no nos delate y lo atamos; desconociendo que "Existen efectivamente distintos tipos de sentimientos que dependen esencialmente del cuerpo" (Von Hildebrand, 2016, p.11). Solo permitimos el movimiento de nuestros ojos para mirar que los estudiantes mantengan la rigidez y sigan tomando nota.

[...] la disciplina escolar (...) tradicionalmente ha puesto en práctica el control de los cuerpos a través de la prescripción de posturas corporales, la vigilancia de la presentación personal y el cuidado por el uniforme; así como también mediante la regulación de la expresión de afectos y los contactos corporales. (Tomasini, 2015, p. 127)

Hemos desconocido un cuerpo que se desequilibra y va perdiendo energía a medida que pasa la jornada escolar, porque nos han enseñado a separarlo de la mente; lo olvidamos y pretendemos que nuestros estudiantes hagan lo mismo, sincronizando su reloj interno y pretendiendo no tener en cuenta esas batallas que se gestan de manera interna en nuestro cuerpo y, por ende, en sus cuerpos también, que muchas veces hacen perder la concentración en el intento de mantener su homeostasis. Es decir, hemos olvidado al estudiante en su humana condición, lo que no permite fluir la emoción en el entre-nos de la relación pedagógica. Desconociendo que las respuestas afectivas (Von Hildebrand, 2012), se expresan "...también en determinadas actitudes corporales y movimientos" (p. 12).

Y pese a estos olvidos, pretendemos que la mente de nuestros estudiantes siga haciendo conexiones con nuestras palabras. Confundimos el silencio con disciplina y nos sentimos satisfechos con esta actitud, desconociendo esa lucha del cuerpo 
y esas miradas que pretenden conmover, pero que no logramos interpretar. Sin embargo, hemos de reconocer que esa disonancia es en la que nos hemos formado, prohibiendo al cuerpo expresar lo que siente.

Siendo así las cosas y como lo plantean los estudiantes, debemos ubicar nuevas cosmovisiones; " ...enseñar exige riesgo, asunción de lo nuevo" (Freire, 2004, p.17), para visibilizar una imagen diferente del cuerpo y un retrato sensible de él mismo, para ahondar en su interior y explorar más allá de lo que conocemos y vemos de nuestros estudiantes; algo que se nos olvidó, algo que perdimos, algo que se nos quitó de las manos. Los estudiantes son más que un cuerpo, son más que un número, son más que un nombre que los identifica, son emociones, son sentimiento, son pensamiento construido en su trasegar, configurado por su historia de vida.

Entender la enseñanza como una formación de la sensibilidad perfila al pedagogo como un esteta social, alguien que tiene como materia prima el cuerpo a fin de modelarlo desde una cierta idealidad, provocando el gesto desde el lenguaje, con el propósito de favorecer la emergencia de sensibilidades y afecciones que tienen como paradigma el acercamiento delicado a la realidad del otro. (Restrepo, 1994, p.45)

Tras esto, al intentar recuperar las emociones, se ha de gestar un cuerpo que se ajuste a las particularidades de la escuela de hoy, con sensaciones divergentes, con manifestaciones impensadas, con absurdos desprovistos, tatuado y permeado por muchas historias colectivas, pero sobretodo particulares y diversas; un cuerpo lleno de emociones que se deprime en el actuar, que se manifiesta en la diversidad, que relata sucesos, que dice sus experiencias, que guarda en el discurso y dice en la expresión; emociones que nos posibilitan estar en el mundo de la vida. Tal como lo expresa Cataño (2017), es necesario pensar una nueva forma de realizar conexiones con los estudiantes, en donde afloren "...los afectos, los sentimientos, las emociones que se expresan en las relaciones intersubjetivas que construyen compartiendo, estando juntos" (p.33).

Lo que se traduce en que dentro de nuestro quehacer debemos empezar a internarnos en la búsqueda de un gran número de estrategias encaminadas a conseguir que nuestros estudiantes se impregnen del nuevo conocimiento, llenas de paradojas, de prácticas de carácter imprevisible, donde nos permitamos sacudir su emocionalidad dormida para comprender las consecuencias a que esto conlleve, deduciendo que no lograremos un efecto ni una transcendencia inmediata en ellos con esta experimentación, sino con la idea de que lo verdaderamente importante 
pueda suceder en cualquier momento del mañana, de tal manera que la huella dejada en ellos a través de las acciones pedagógicas coadyuven en su desempeño dentro de su realidad contextual.

\section{Discusión}

Escuchar a los estudiantes fue un espacio valioso para tomar distancia y alejarnos de una perspectiva adultocéntrica que es resistente y no permite tener una idea clara de las vivencias de los estudiantes, quienes nos exhortan a comprender la importancia del rol de las emociones puesto en el escenario de la escuela y reconocer en qué medida hemos podado y castrado bajo directrices institucionales a un cuerpo que las hace perceptibles, las gestualiza y las verbaliza.

El contexto educativo actual, viene transformándose de manera vertiginosa y permanente, lo que genera interrogantes respecto de las problemáticas que están emergiendo en los espacios educativos. Se advierte un gran enfrentamiento entre los sentidos de los tiempos generacionales, los cuales cada vez son más diversificados que articulados en cuanto a la relación entre la asignación y asimilación del saber en medio de los sujetos de la educación. La distancia entre maestro y estudiante, produce fragmentaciones y fisuras en la relaciones.

Los estudiantes manifiestan que dichas fisuras y fragmentaciones, en su mayoría, vienen dadas por la pretensión del maestro de seguir enseñando desde la homogeneidad. Sigue siendo constante por parte de algunos maestros, una postura de resistencia o tal vez de impotencia frente a estos cambios que están emergiendo, en tanto alteran su cotidianidad. "El normalismo (una práctica demasiado extendida en la sociedad contemporánea) ha provocado daños irreparables, ya que ha sido el patrón que ha regido lo que está permitido y lo que no" (Planella, 2018, p. 76).

Para algunos maestros es dificil entender que gran parte de los estudiantes han nacido y crecido en tiempos de grandes mutaciones, tanto culturales como comunicacionales, donde sus relaciones son más del tipo digital e interactivo; por tanto, su mentalidad y modos de ver la vida son totalmente diferentes a las del maestro, quien en su tiempo, las relaciones y las pautas de crianza estaban mediadas por la familia, la escuela, la iglesia y las calles fueron los escenarios para compartir experiencias. 
Todas estas circunstancias siguen marcando una gran distancia en la relación pedagógica. Aunado a ello, encontramos en las aulas estudiantes que no se quieren quedar en un solo lugar, que tienen la opción de conocer el mundo de otras maneras, de comunicarse en el acto con personas de otros países. Mientras tanto, los maestros vivimos otros tipos de experiencias, puesto que somos de otra generación. Todo lo tenemos planeado, sistematizado; todo es cíclico. Muchos maestros siguen viviendo en su tiempo y en su estructura, sin querer entender que el estudiante viene y ha crecido en otras lógicas.

Es claro para la mayoría que una cosa es el tiempo generacional del maestro de los años 60, 70 u 80, quien aprendió de memoria y bajo parámetros de una educación tradicional y otra muy distinta es el tiempo generacional de los estudiantes que nacieron en las décadas de los 90 y 2000; son tiempos y necesidades totalmente diferentes. El estudiante que encontramos en los espacios educativos es ajeno a la monotonía, a la norma, a la parametrización, a la psicorigidez que venía marcando la educación tradicional. Es una ardua tarea por emprender, la de comprender estas nueva lógicas y demandas.

Es relevante que los maestros tomemos conciencia de las transformaciones que se vienen dando en nuestra realidad circundante, pero que al mismo tiempo seamos capaces de interpretar esas variaciones y asumir nuestra responsabilidad y compromiso para reflexionar sobre los fenómenos sociales, culturales, tecnológicos, políticos y económicos que acontecen en la actualidad, pero sin dejarnos arrastrar por ellos. Es decir, tener apertura a nuevos contextos que inevitablemente permean la escuela.

Con base en lo anterior, estamos obligados a dar lo que no está en internet, la parte humana, la parte sensible; nuestro saber, nuestro hacer y nuestro sentir, complementados con la entrega de nuestro ser; estamos además obligados a hacer conexiones de empatía con los estudiantes para llevarlos a crecer, a sensibilizarse y a convivir. Sin embargo, la empatía no es suficiente, puesto que si no reconocemos al otro en su singularidad, en su diversidad y en su particularidad y mientras no demos espacios para asimilar que todos tenemos cosmovisiones distintas de la realidad, la empatía se rompe y da entrada al conflicto.

La conflictividad que implica el aula, tanto desde el punto de vista de las exigencias de estudio y preparación como de organización y conducción de los saberes que en ella se mueven, no puede ser minimizada en favor de una "comunicación y apoyo afectivo" de estilo asistencial, porque se perdería lo esencial del acto pedagógico, que es enseñary aprender un contenido específico de una disciplina, un valor o una norma de convivencia. (Bertoni, 2015, p. 24) 
De esta manera, no solamente nos encontramos frente a desafíos valorativos, hemos de comprender el presente de una generación en relación al presente de otra muy diferente. Para algunos de nosotros el tiempo puede ser lentitud, para los estudiantes de ahora es vértigo, es afán, es aventura, es hacer varias cosas al tiempo. La realidad entonces nos plantea ajustarnos a esas nuevas lógicas que están emergiendo, resignificando la relación pedagógica; para ello no es suficiente tener apertura para descifrar simbologías que se van presentando en los contextos, sino además dejar a la emoción fluir en el entre-nos, lo que nos posibilitará potenciar nuevas formas de interacción y orientación al otro, ese estudiante que se encuentra junto a nosotros y con quien compartimos tanto tiempo al día.

Conviene recalcar que los espacios de vivencia diaria con el estudiante, donde como maestros ponemos en juego múltiples aspectos que al confluir pueden potenciar el logro o fracaso de la relación pedagógica y por ende de los procesos pedagógicos que allí desarrollamos, son el resultado de la convergencia de varios aspectos que actúan simultáneamente y se conjugan para alcanzar como resultado la gestación de nuevas formas de aprender dentro de la diversidad de opciones y de sujetos que se encuentran en dicho entorno. Aún no somos conscientes que los problemas, más que pensarlos desde ajustes a la normatividad o al mismo sistema educativo, tienen su raíz en el sujeto y su papel preponderante en la sociedad; es una época de tensiones y transformaciones que nos exige como maestros considerar una lectura diferente de mundo a la que veníamos haciendo.

\section{Conclusiones}

La resignificación de la relación pedagógica ha de basarse en varios asuntos, uno de ellos implica de manera indiscutible y a solicitud expresa de los estudiantes, dejar a la emoción fluir en el entre-nos de la escuela; para ello, es perentorio que el maestro realice un ejercicio reflexivo acerca de "...su hacer pedagógico y así, modificar y transformar a los sujetos que tiene a cargo; el maestro de hoy debe forjar actitudes políticas, éticas y sociales en sus estudiantes" (Zapata, 2011, p. 64). Esto involucra propender por una pedagogía sensible que potencie en la vivencia cotidiana, el ejercicio práctico de las dimensiones emocional y afectiva, a partir de:

- El actuar comunicacional: es decir, comprender la diversidad, respetarla y aceptarla a partir de acuerdos y consensos. Se hace necesario formarnos en la capacidad de deliberación para solucionar no solo los conflictos escolares, que mejoraría sin duda alguna la relación pedagógica, sino que además 
aportaría a la resolución de los conflictos sociales en que todos nos vemos envueltos en nuestro día a día, especialmente nuestros estudiantes, para que actúen reflexiva y críticamente en la toma de decisiones de manera consciente y responsable.

- El pluralismo razonable: reconocer al otro en la diferencia estableciendo el diálogo con calidad argumentativa para convalidar la complementariedad y la reciprocidad dentro de la comunidad escolar. Una actitud de escucha atenta y activa durante espacios de real conversación fuera de lo académico, para dar importancia a lo que el estudiante expresa como interloculor válido.

- La cultura de la diversidad: potenciar una educación en valores y en virtudes desde donde se propenda por reconciliar lo que se dice, con lo que se piensa y se hace. Una coherencia de la relación pedagógica que en lugar de generar sentimientos de indignación, propicie la solidaridad y responsabilidad ética hacia el otro.

\section{BIBLIOGRAFÍA}

Álvarez, M. (2012). La Investigación Biográfica, en el marco de la investigación cualitativa. CONHISREMI, 8(3), 53-76. Recuperado de https://bit.ly/2F4kyRt

Aramburo, J. y Vargas, N. (2015). El maestro y la maestra en relación pedagógica: comprensión de seres humanos actantes en la escuela, en una mirada de sí mismo, del otro y de lo otro. (Tesis de Maestría). Recuperado de https:// bit.ly/2Fj9vHx

Bedoya, N. y González, M. (2016). Las soledades en una mujer docente: reflexiones de un relato autobiográfico. Anduli, Andaluza de Ciencias Sociales, (15), 119-138. Recuperado de https://bit.ly/2SKGZBV

Bertoni, E. (2015). La dimensión afectiva e intersubjetiva del aprendizaje. Una mirada desde la filosofía. InterCambios, 2(1), 21-26. Recuperado de https://bit.ly/2Foul7V

Bolívar, A., Domingo, J. y Fernández, M. (2001). La investigación biográfico-narrativa en educación. Enfoque y metodología. Madrid, España: La Muralla.

Cadavid, A. y Parra, J. (2010). La autorregulación de los errores en las evaluaciones escritas de niños y niñas en la ciudad de Manizales. Plumilla Educativa, (7), 129-144. Manizales: Universidad de Manizales.

Cataño, M. (2017). Parque de los afectos: comunicación, modos de estar juntos y reconocimiento en las relaciones intersubjetivas de jóvenes en la experiencia de habitar un parque. Tesis de Doctorado. Recuperado de https://bit.ly/2QCaqEu

Freinet, C. (2005). Tecnicas Freinet de la escuela moderna. (36ª ed.). México: Siglo XXI. 
Freire, P. (2004). Pedagogía de la Autonomía. Saberes necesarios para la práctica educativa. Sao Paulo: Paz e Terra S.A.

García, A. y Sabido, O. (2017). El estudio sociológico del amor corporeizado: la construcción de un objeto de estudio entrelazando teorías y niveles analíticos. Estudios Sociológicos, 35(105), 653-675. Recuperado de https://bit.ly/2H89/Vh

Goleman, D. (2009). Inteligencia emocional. (20a ed.). España: Kairós.

Granada, D. (2015). Emociones y toma de decisiones éticas: implicaciones y desarrollo para la empresa. Bogotá, Colombia: Universidad del Rosario.

Jelin, E. (2014). Las múltiples temporalidades del testimonio: el pasado vivido y sus legados presentes. Clepsidra, 1(1), 140, 163. Recuperado de https://bit.ly/2VGzC02

López, S. (2010). Maestros en el territorio. La Serena, Chile: Universidad de la Serena.

Peralta, M. (2008). El derecho de los más pequeños a una pedagogía de las oportunidades en el siglo XXI. Revista Iberoamericana de Educación, 47, 33-47. Recuperado de 47, 33-47. Recuperado de https://bit.ly/2TzMbbl

Planella, J. (2018). Pedagogías sensibles. Sabores y saberes del cuerpo y la educación. Barcelona, España: Universidad de Barcelona.

Restrepo, L. (1994). El derecho a la ternura. Bogotá: Arango editores.

Tomasini, M. (2015). Feminidades juveniles, cuerpos y producciones estéticas en la escuela secundaria. Revista Iberoamericana de Educación, 68, 117-136. Recuperado de https://bit.ly/2TAb4UO

Villegas, M. y González, F. (2011). La investigación cualitativa de la vida cotidiana. Medio para la construcción de conocimiento sobre lo social a partir de lo individual. Psicoperspectivas, 10(2), 35-59. Recuperado de https://bit.ly/2QAQAth

Von Hildebrand, D. (2016). Las formas espirituales de la afectividad. Madrid, España: Encuentro.

Zemelman, H. (2005). Voluntad de conocer: el sujeto y su pensamiento en el paradigma crítico. Barcelona, España: Anthropos. 